\title{
TILLAGE TIME AFFECTS SOIL HYDRO-THERMAL PROPERTIES, SEEDLING GROWTH AND YIELD OF MAIZE (ZEA MAYS L.)
}

\author{
WANG, X. - Yue, Y. - NOOR, M. A. - Hou, H. - ZhOU, B. - MA, W.*-ZHAO, M.* \\ Institute of Crop Sciences, Chinese Academy of Agricultural Sciences, Key Laboratory of Crop \\ Physiology and Ecology, Ministry of Agriculture, 100081 Beijing, P. R. China \\ *Corresponding authors \\ e-mail:mawei02@caas.cn, zhaoming@caas.cn; phonelfax: +86-10-8210-8752 \\ (Received $9^{\text {th }}$ May 2018; accepted $11^{\text {th }}$ Jul 2018)
}

\begin{abstract}
Two-year experiments were conducted to determine optimum tillage time to improve soil compaction and hydro-thermal properties for maize growth. Two tillage treatments: no-tillage (NT), and subsoiling (SS) were performed in correspondence to eight tillage times from March 25 to July 20 (I: March 25, II: April 10, III: April 25, IV: May 10, V: May 25, VI: June 10, VII: July 5, and VIII: July 20) at 15 days interval, synchronously with eight sowing times. SS significantly decreased soil bulk density $(0-30 \mathrm{~cm})$ by about $0.13 \mathrm{~g} \mathrm{~cm}^{-3}$ at times of I-V, and about $0.08 \mathrm{~g} \mathrm{~cm}^{-3}$ at VI-VIII. Increment in total soil porosity due to SS was about $3.88 \%$ during I-V and about $2.27 \%$ at VI-VIII. SS significantly increased soil moisture content by $1.60 \%$ in $10-30 \mathrm{~cm}$ soil profile. Soil minimum temperature significantly increased by about $0.58{ }^{\circ} \mathrm{C}(0-20 \mathrm{~cm})$ for $\mathrm{I}-\mathrm{V}$, while an increase of $1.06{ }^{\circ} \mathrm{C}(0-10 \mathrm{~cm})$ was seen for maximum soil temperature due to SS. Greater seedling dry matter coupled with higher growth, and yield improvement was achieved with SS. Dry matter accumulation and grain yield was maximum for May 25 to June 10 sowing as compared to early $(22 \%, 43.5 \%)$ and late $(60 \%, 41 \%$, respectively) sowing due to SS versus NT. Initial SS intervals could alleviate soil compaction and low temperature stress for spring maize, with improved soil hydrothermal properties.
\end{abstract}

Keywords: subsoiling time, soil bulk density, soil moisture, soil temperature, maize growth

\section{Introduction}

No-tillage in maize (Zea mays L.) is the dominant agricultural practice in HuangHuai-Hai $(\mathrm{HHH})$ maize growing region in China, and the long-term no-tillage is often unfavorable for the growth of maize. Soil compaction has been a major limiting factor for soil environment and plant growth (Zheng and Yan, 2006). To improve soil environment and health, subsoiling tillage as an effective measure is getting popular for maize growth ( $\mathrm{Ji}$ et al., 2013). Normally, soil tillage and seed sowing are always conducted in June with low precipitation and high temperature in $\mathrm{HHH}$ region. Although, no-tillage is practiced for maize sowing in $\mathrm{HHH}$ region, except a little soil disturbance at the time of sowing, under winter-wheat and summer-maize cropping system. However, intensive tillage could cause serious soil surface water evaporation during this period, especially with high temperature ( $\mathrm{Yu}$ and Zhang, 2005), and intensive tillage over longer periods also alters the chemical and physical properties of soil (Lozano-García and Parras-Alcántara, 2014; Jabro et al., 2009). This could lead to a severe drought stress for maize seedling growth (Zhou et al., 2009). Especially under the recent scenario of climate change, in which air temperature is increasing and causing irregular rainfall flashes in northern China (Zafar et al., 2018; Piao et al., 2010; Guo et al., 2010). To avoid the negative effects of soil problems and external micro and macro climate on maize growth and development, the time of soil tillage and sowing date considering the external environment should be given greater attentions (Noor, 
2017; Yin et al., 2017). Therefore, identifying an effective tillage time to decrease soil compaction level and improving soil hydro-thermal properties is very important for the maize seedling growth (Nath and Lal, 2017; Lu et al., 2016).

Recently, great progress has been made through soil tillage management in reducing soil bulk density and increasing soil moisture storage. Subsoiling tillage is reported as conservation tillage practice, by improvement of soil characteristics for maize sowing and seedling growth (Liu et al., 2009; Hou et al., 2012; Hoffmann and Kismányoky, 2001). Many studies indicated that subsoil deep tillage could break the soil plough pan and reduces soil bulk density to a proper soil compaction in northeast China (Wang et al., 2014). Soil structure and hydro-thermal characteristics are not only influenced by soil tillage management, but also highly sensitive to the external environmental conditions such as temperature and precipitation (Karandish and Shahnazari, 2016; Hartmann et al., 2012; Bhaduri et al., 2017).

Therefore, studying the effects of subsoiling tillage implemented at different time intervals has practical guiding value for selecting optimal soil tillage management in maize. Our objectives in present study were, in the process of soil improvement, (1) to find the differential effects of subsoiling tillage on soil compaction and hydro-thermal properties at different subsoiling tillage times; (2) to evaluate the specific subsoiling tillage time alleviating the soil compaction stress at maize seedling growth; (3) to identify optimum time for subsoiling tillage for improving soil compaction problem and minimizing drought stress effects encountered by maize at the seedling stage in $\mathrm{HHH}$ region.

\section{Materials and methods}

\section{Site description}

The field experiments were conducted in Xinxiang Experiment Station of Chinese Academy of Agricultural Sciences, Henan Province, China (35¹8áN, 11354áE). Soil textural class for studied site is classified as clay loam (ISSS Classification, International Soil Science Society), with percentage composition of sand, silt and clay as $32.95,34.2$ and $32.85 \%$, respectively (saturation: $38.5 \%$ ). Soil bulk density was $1.29 \mathrm{~g} \mathrm{~cm}^{-3}, 1.34 \mathrm{~g} \mathrm{~cm}^{-3}, 1.55 \mathrm{~g} \mathrm{~cm}^{-3}$ and $1.52 \mathrm{~g} \mathrm{~cm}^{-3}$ at $0-10 \mathrm{~cm}, 10-20 \mathrm{~cm}, 20-30 \mathrm{~cm}$ and $30-40 \mathrm{~cm}$ soil depth, respectively. Major soil nutrient contents analyzed were: organic matter $12.55 \mathrm{~g} \mathrm{~kg}^{-1}$, total nitrogen $1.13 \mathrm{~g} \mathrm{~kg}^{-1}$, available phosphorus $16.15 \mathrm{mg}$ $\mathrm{kg}^{-1}$, and $109.95 \mathrm{mg} \mathrm{kg}^{-1}$ available potassium $\left(\mathrm{pH}=8.21, \mathrm{EC}=1.2 \mathrm{dSm}^{-1}\right.$, slope gradient with negligible risk of soil erosion). Usual crop rotation for this area is double cropping system with winter-wheat and summer-maize, in that rotary tillage $(15-20 \mathrm{~cm})$ is practiced for wheat and no-tillage farming for maize crop is followed usually.

Annual mean temperature and accumulated precipitation conditions from March to July during 1990 to 2012 are shown in Figure 1. From year 1990 to 2012, annual accumulated precipitation had a decline trend, whereas annual mean temperature gradually increased.

\section{Experimental design and soil tillage management}

The experiment was conducted from 2011 to 2012. Randomized complete block design was used with split plot arrangement and three replications. Main plots were assigned to tillage times correspondingly with eight sowing intervals starting from 
March 25 to July 20 (I: March 25, II: April 10, III: April 25, IV: May 10, V: May 25, VI: June 10, VII: July 5, and VIII: July 20). Whereas, in subplots two soil tillage treatments just before the maize sowing were conducted viz. SS: subsoiling tillage management involved complete soil inversion and burial of crop residues to a depth of $30 \mathrm{~cm}$ using a stripe deep loosening machine (Hehuinong machine Co. Ltd., Beijing, China)., NT: no tillage for two consecutive years. The experimental plots were $30 \mathrm{~m}$ long and $3.6 \mathrm{~m}$ wide and consisted of 6 rows. Maize sowing and soil tillage were conducted at the same day, so the experiment had eight sowing dates correspondingly. There was no deep soil tillage management for the rotational crops and the plough pan lies at the depth of $20-30 \mathrm{~cm}$ of soil profile. The maize variety Zhengdan-958 was planted at the density of 67500 plants $^{-1}$ with an 80-40 $\mathrm{cm}$ wide/narrow row spacing pattern. $\mathrm{N}$ fertilizers at $225 \mathrm{~kg} \mathrm{~N}^{-1}$ (Urea: $46 \% \mathrm{~N}$ ) were applied in two split applications: $1 / 3$ as basal application before planting, and the remaining $2 / 3$ as sidedressing at the V8-V9 growth stage. Phosphate $\left(\mathrm{P}_{2} \mathrm{O}_{5}\right)$ and Potassium $\left(\mathrm{K}_{2} \mathrm{O}\right)$ fertilizers were applied at $75 \mathrm{~kg} / \mathrm{ha}$ and $150 \mathrm{~kg} /$ ha before sowing, respectively.

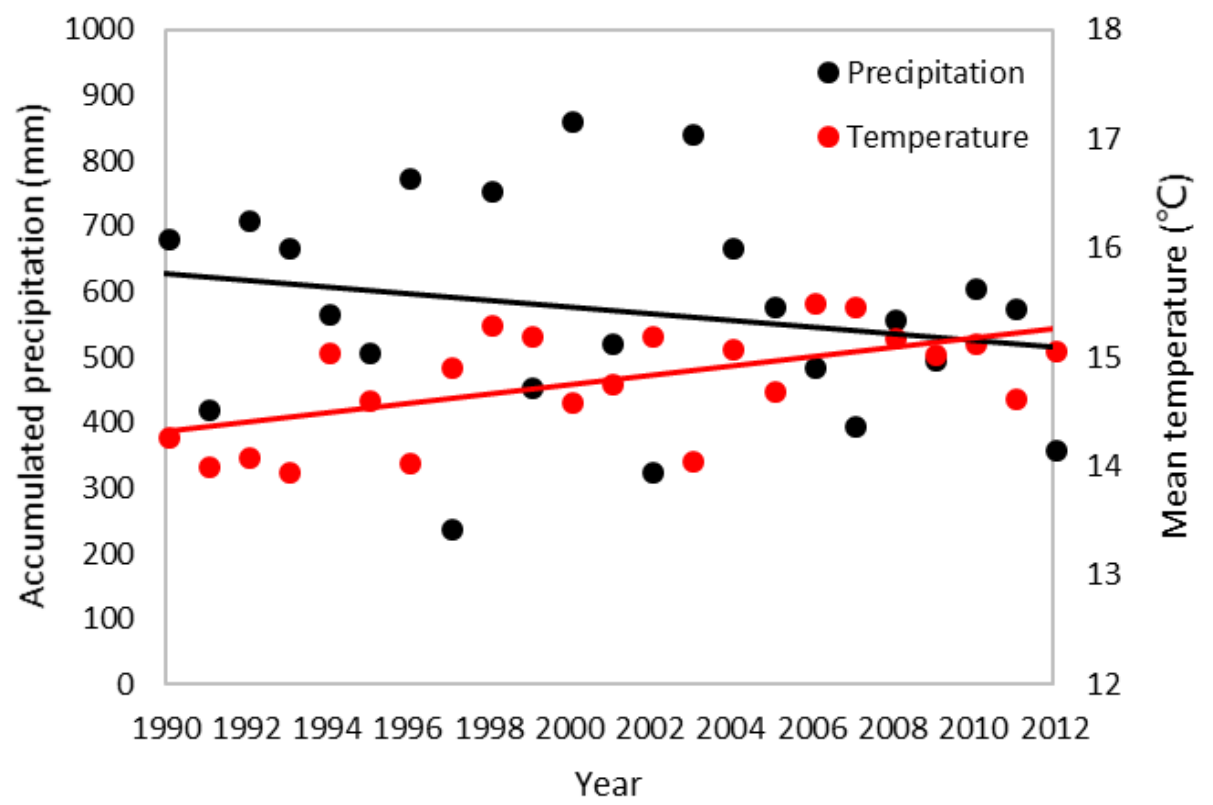

Figure 1. Changes of annual mean temperature and accumulated precipitation from 1990 to 2012

\section{Sampling and measurement}

Soil samples were collected from four soil depths $(0-10 \mathrm{~cm}, 10-20 \mathrm{~cm}, 20-30 \mathrm{~cm}$, and $30-40 \mathrm{~cm}$ ) for each tillage treatment 20 days after soil tillage implementation. In each plot, soil sample was obtained to determine soil bulk density, soil porosity, soil moisture content and soil temperature. For plant samples plant shoots were cut manually at the ground level to determine the shoot dry weight, and plant roots were dug out from soil profile of $40 \mathrm{~cm}$ depth to determine the root dry weight for 3 plants per plot at 20 days after soil tillage management.

Soil bulk density was determined by weighing method $(E q .1)$ with cutting-ring which had a fixed volume of $100 \mathrm{~cm}^{3}$ to sample the soil maintaining the soil physical structure at the same time. Then weighing the soil fresh weight and dry weight: 


$$
\text { Soil bulk density }\left(\mathrm{g} / \mathrm{cm}^{3}\right)=\frac{\text { soil dry weight }(\mathrm{g})}{\text { cutting-ring volume }\left(\mathrm{cm}^{3}\right)}
$$

The measurement was made at four depths of soil (0-10, 10-20, 20-30, and 30$40 \mathrm{~cm})$ at seedling stage of maize. The measurement was repeated three times in each plot. Total soil porosity was calculated (Eq. 2) from soil bulk density as follows:

$$
\text { Total soil porosity }(100 \%)=\frac{(\text { soil density }- \text { soil bulk density) }}{\text { soil density }}
$$

where soil density is $2.65 \mathrm{~g} \mathrm{~cm}^{-3}$. For soil moisture content (Eq. 3), soil samples were taken from the $0-40 \mathrm{~cm}$ soil profile at $10 \mathrm{~cm}$ intervals and oven-dried to constant weight in an aluminum container.

$$
\text { Soil moisture content }(100 \%)=\frac{\text { (fresh soil weight }- \text { dry soil weight) }}{\text { dry soil weight }}
$$

The measurement was repeated three times in each plot at 20 days after sowing of maize. For soil temperature measurement, thermistor temperature probes were buried at three points in each plot and four depths in the $0-20 \mathrm{~cm}$ soil profile $(0-5,5-10,10-15$, and $15-20 \mathrm{~cm}$ ). Daily soil minimum and maximum temperatures were measured at consecutive 20 days after sowing of maize.

Sampling was done from each plot by cutting three adjacent plants in a row at ground level to determine leaf area and dry matter accumulation at V3, V6, VT and R3 growth stages. Leaf area index (LAI) was calculated by multiplying leaf area with plant density per $\mathrm{m}^{2}$. Already sampled three plants were oven dried at $105{ }^{\circ} \mathrm{C}$ for one hour initially and then at $71 \pm 1{ }^{\circ} \mathrm{C}$ to measure dry matter accumulation. Twenty days after sowing of maize, five whole plants were randomly sampled from each sub-plot and then plant shoot and roots were separated and dry matter was determined. At harvest stage, maize ears were hand harvested in the two central rows (the length of each row was $20 \mathrm{~m}$ ) of each plot, dried and shelled. Dry matter and yields were obtained according to standard procedures. The maize yield potential (YP) in HHH area was obtained by the HybridMaize simulation model (Yang et al., 2004).

The effect of soil tillage time on all analyzed parameters was tested using a one-way analysis of variance (ANOVA) (SPSS 13.0 for Windows; SPSS Inc., Chicago, USA). Tukey's HSD method was used as post hoc test for multiple comparisons of means. Mean data was presented for both years (2011 and 2012) for all the studied parameters except for some growth attributes (LAI, dry matter accumulation), due to nonsignificant year effect for the studied period.

\section{Results}

\section{Soil and external environment before soil tillage}

The values of soil temperature and soil moisture were significantly lower at initial soil tillage time intervals than later tillage intervals (Table 1). Soil mean temperature at different soil tillage times were recorded as the sequence of I $<$ II $<$ III $<$ IV $<$ V $<$ VI $<$ VII $<$ VIII. 
Table 1. Soil hydrothermal properties before soil tillage in $0-40 \mathrm{~cm}$ soil depth

\begin{tabular}{c|c|c|c|c|c|c|c|c}
\hline Soil tillage time & I & II & III & IV & V & VI & VII & VIII \\
\hline Soil mean temperature $\left({ }^{\circ} \mathrm{C}\right)$ & 16.53 & 17.23 & 18.52 & 22.39 & 24.60 & 25.63 & 29.53 & 30.00 \\
Soil mean moisture content $(\%)$ & 14.14 & 14.47 & 15.15 & 15.84 & 16.56 & 16.66 & 15.87 & 17.14 \\
Soil mean bulk density $\left(\mathrm{g} \mathrm{cm}^{-3}\right)$ & 1.36 & 1.35 & 1.36 & 1.32 & 1.39 & 1.31 & 1.37 & 1.37 \\
\hline
\end{tabular}

I: March 25, II: April 10, III: April 25, IV: May 10, V: May 25, VI: June 10, VII: July 5, VIII: July 20

The values of soil mean moisture content at different soil tillage times were recorded in the sequence of $\mathrm{I}<\mathrm{II}<\mathrm{III}<\mathrm{IV}<\mathrm{VII}<\mathrm{V}<\mathrm{VI}<\mathrm{VIII}$. The values of effective accumulated temperature for different soil tillage times were as the sequence of IV $>$ III $>$ V $>$ II $>$ VI $>$ VII $>$ I $>$ VIII during whole crop growth period (Fig. 2).

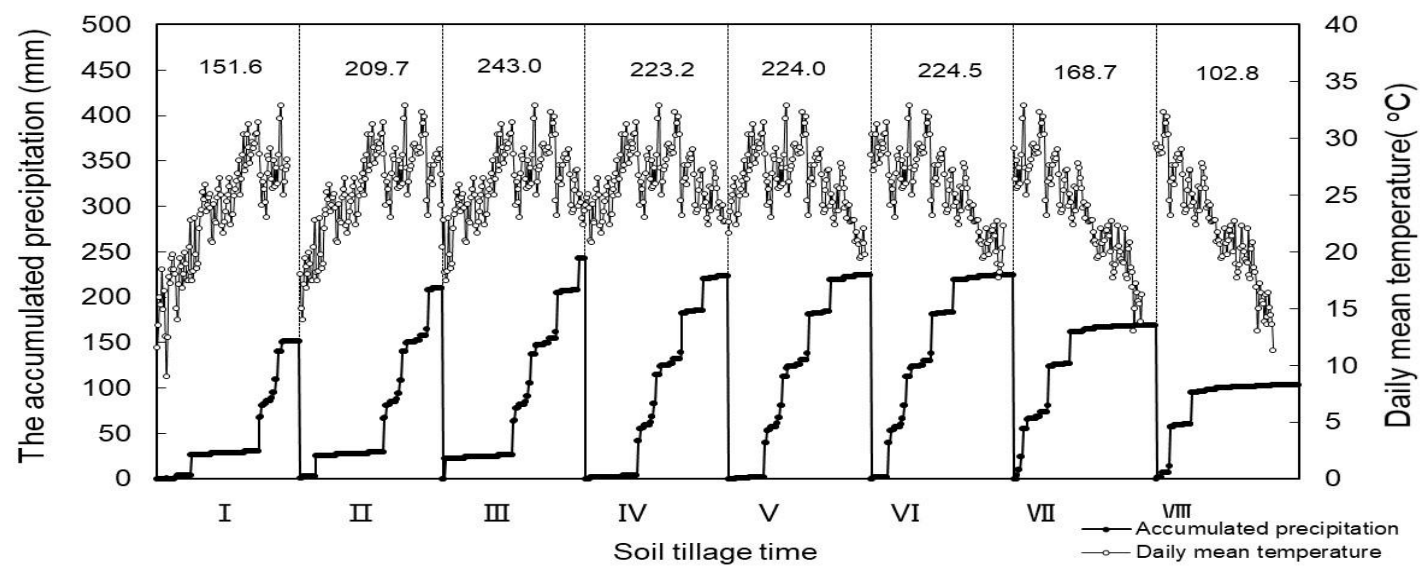

Figure 2. Effective accumulated temperature (top word in figure), daily mean temperature (center line in figure) and accumulated precipitation (bottom line in figure) during growth cycle of maize at different soil tillage time. I: March 25, II: April 10, III: April 25, IV: May 10, V:

May 25, VI: June 10, VII: July 5, and VIII: July 20

\section{Soil bulk density}

In 0-10 cm soil layer, SS significantly decreased soil bulk density by about $0.13 \mathrm{~g} \mathrm{~cm}^{-3}$ $(\mathrm{P}<0.01)$ at former tillage time of $\mathrm{I}-\mathrm{V}$, and the decrease was about $0.04 \mathrm{~g} \mathrm{~cm}^{-3}(\mathrm{P}<0.05)$ at later soil tillage intervals of VI-VIII, compared to NT (Fig. 3). In 10-20 cm soil layer, soil bulk density significantly decreased by about $0.07 \mathrm{~g} \mathrm{~cm}^{-3}(\mathrm{P}<0.05)$ at each soil tillage time under SS treatment compared to NT. Because of the destruction of soil hard plough pan, SS significantly decreased soil bulk density by about $0.15 \mathrm{~g} \mathrm{~cm}^{-3}(\mathrm{P}<0.01)$ at each soil tillage time compared to NT in $20-30 \mathrm{~cm}$ soil layer.

\section{Soil porosity}

Because of the decrease in soil bulk density, soil total porosity was therefore increased significantly (Fig. 4). In 0-10 cm soil layer, SS significantly increased soil total porosity by $4.18 \%(\mathrm{P}<0.01)$ at initial tillage intervals of $\mathrm{I}-\mathrm{V}$, and the increment was $2.38 \%$ $(\mathrm{P}<0.05)$ at later soil tillage time of VI-VIII compared to NT. SS significantly increased soil porosity with average of $3.75 \%(\mathrm{P}<0.01)$ in top $(0-30 \mathrm{~cm})$ soil layer at initial tillage intervals of $\mathrm{I}-\mathrm{V}$, while the increment was $2.26 \%(\mathrm{P}<0.05)$ at tillage intervals VI-VIII, as compared to NT. 

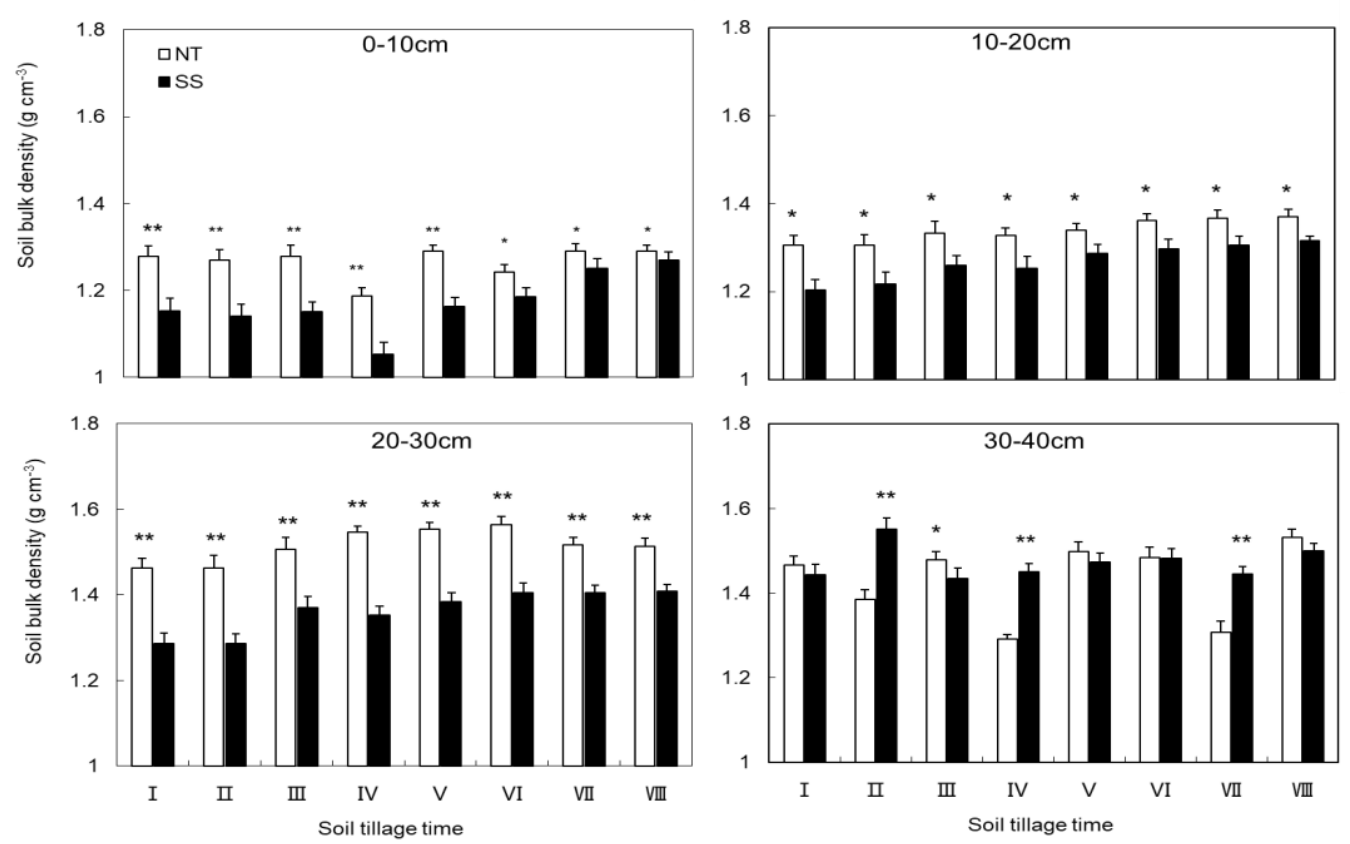

Figure 3. Soil bulk density in 0-40 cm depth at different soil tillage time. NT: no tillage, SS: subsoiling tillage; I: March 25, II: April 10, III: April 25, IV: May 10, V: May 25, VI: June 10,

VII: July 5, and VIII: July 20; ** above the column indicate statistical significance at the $P=0.01$ level within the same soil tillage time; $*$ above the column indicate statistical significance at the $P=0.05$ level within the same soil tillage time
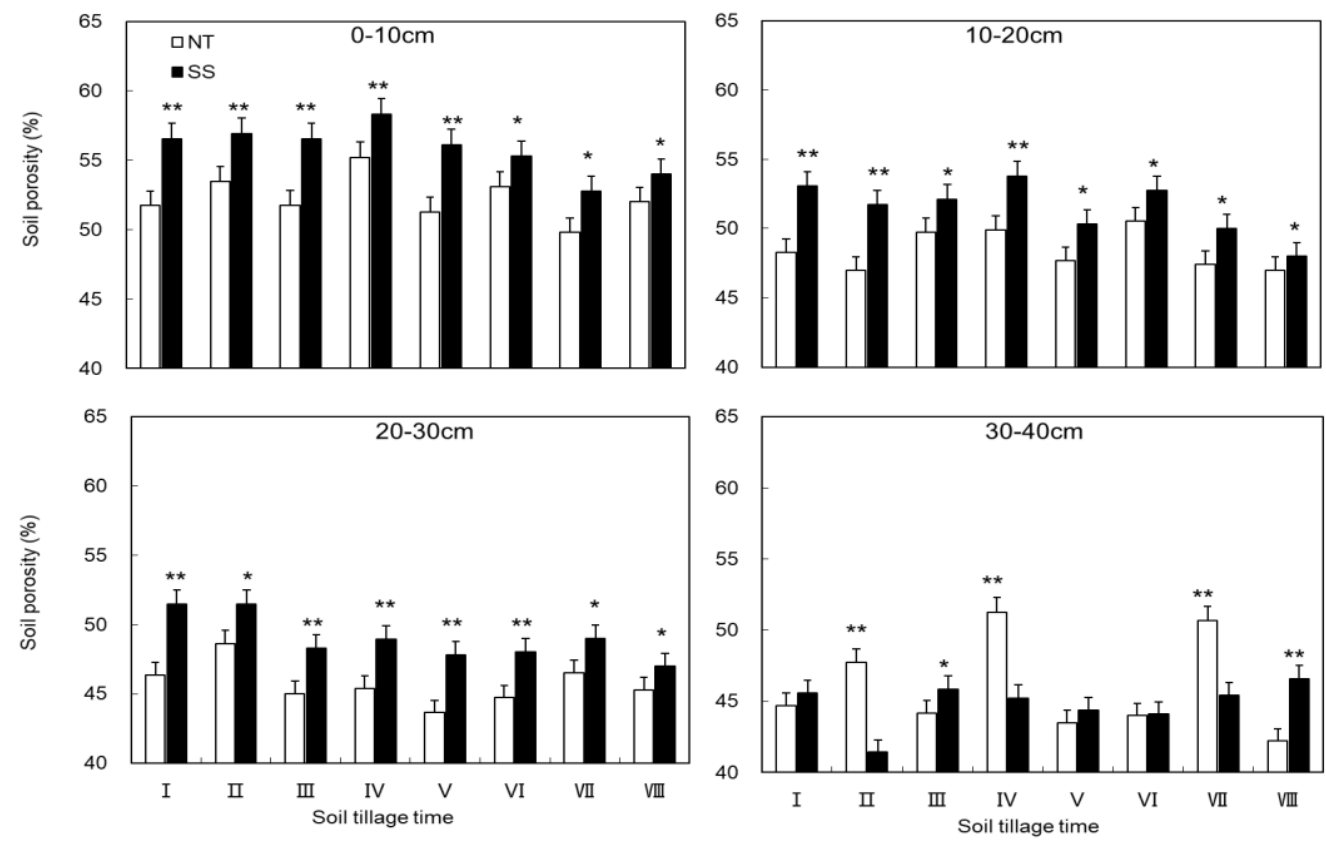

Figure 4. Soil porosity in 0-40 cm depth at different soil tillage times. NT: no tillage, SS: subsoiling tillage; I: March 25, II: April 10, III: April 25, IV: May 10, V: May 25, VI: June 10, VII: July 5, and VIII: July 20; ** above the column indicate statistical significance at the $P=0.01$ level within the same soil tillage time; $*$ above the column indicate statistical significance at the $P=0.05$ level within the same soil tillage time 


\section{Soil moisture content}

In $0-10 \mathrm{~cm}$ soil layer, SS significantly decreased soil moisture content by $2.06 \%$ $(\mathrm{P}<0.01)$ at later soil tillage time of VI-VIII, because of the soil disturbance coincidence with high soil evaporation (Fig. 5). In $10-20 \mathrm{~cm}$ soil layer, SS significantly increased soil moisture content by $2.24 \%(\mathrm{P}<0.01)$ at intervals I, II, III, $\mathrm{V}$, and the increment was $1.13 \%(\mathrm{P}<0.05)$ at later intervals of IV, VI, VII, VIII. In $20-30 \mathrm{~cm}$ soil layer, soil moisture content significantly increased by $2.26 \%(\mathrm{P}<0.01)$ at I, II, V, VI intervals, and the increment was about $1.13 \%(\mathrm{P}<0.05)$ at later intervals of III, IV, VII, VIII.
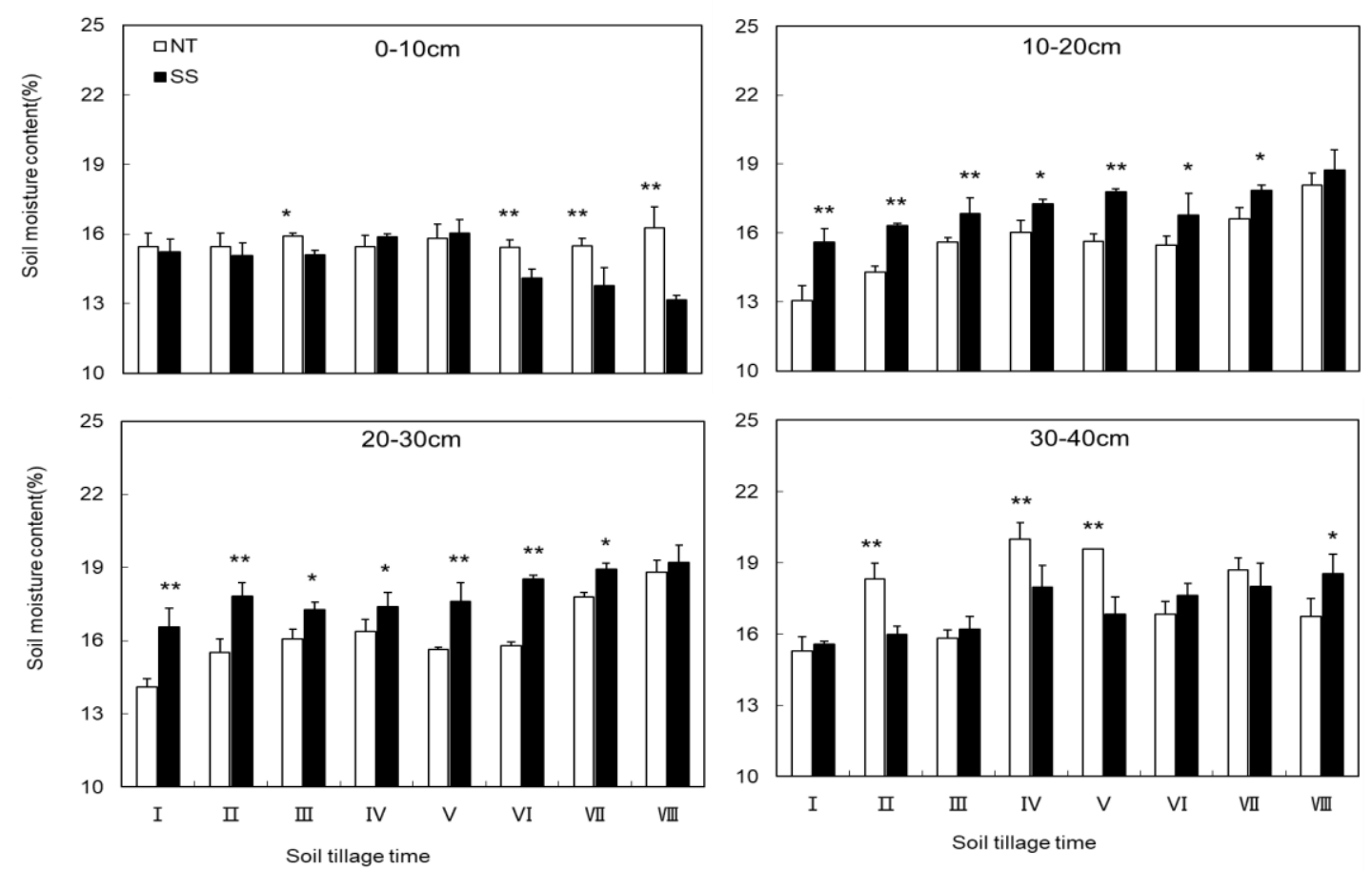

Figure 5. Soil moisture content in 0-40 cm depth at different soil tillage. NT: no tillage, SS: subsoiling tillage; I: March 25, II: April 10, III: April 25, IV: May 10, V: May 25, VI: June 10, VII: July 5, and VIII: July 20; ** above the column indicate statistical significance at the $P=0.01$ level within the same soil tillage time; * above the column indicate statistical significance at the $P=0.05$ level within the same soil tillage time

\section{Soil temperature}

In $0-10 \mathrm{~cm}$ soil layer, $\mathrm{SS}$ was able to increase soil minimum, maximum and mean temperatures as compared to NT (Table 2). For soil lowest temperature, the significant increment was $1.29{ }^{\circ} \mathrm{C}(\mathrm{P}<0.05)$ at tillage time of I, II, while it was $0.51{ }^{\circ} \mathrm{C}$ at intervals III, IV, V, VI. For soil maximum temperature, the increment was about $1.06{ }^{\circ} \mathrm{C}(\mathrm{P}<0.05)$ at each soil tillage time. For soil mean temperature, the increment recorded was $1.12{ }^{\circ} \mathrm{C}(\mathrm{P}<0.05)$ at former soil tillage intervals of I, II, while it was $0.65^{\circ} \mathrm{C}$ at intervals III, IV, V, VI, VII, VIII. In 10-20 cm soil layer, SS just increased soil lowest temperature compared to $\mathrm{NT}$, the increment was $0.43{ }^{\circ} \mathrm{C}$ for tillage intervals of $\mathrm{I}-\mathrm{V}$, while it was $-0.28{ }^{\circ} \mathrm{C}$ at later soil tillage time of VI-VIII, as compared to NT. 
Table 2. Soil minimum, maximum and mean temperature in 0-20 cm soil depth at 20 days after sowing of maize

\begin{tabular}{|c|c|c|c|c|c|c|c|c|c|c|}
\hline \multirow{2}{*}{$\begin{array}{c}\text { Soil } \\
\text { depth } \\
(\mathbf{c m})\end{array}$} & \multirow{2}{*}{$\begin{array}{c}\text { Soil } \\
\text { temperature } \\
\left({ }^{\circ} \mathrm{C}\right)\end{array}$} & \multirow{2}{*}{$\begin{array}{c}\text { Soil } \\
\text { tillage } \\
\text { method }\end{array}$} & \multicolumn{8}{|c|}{ Soil tillage time } \\
\hline & & & I & II & III & IV & $\mathbf{V}$ & VI & VII & VIII \\
\hline \multirow{6}{*}{$0-5$} & \multirow{2}{*}{ Minimum } & NT & $14.71 \mathrm{~b}$ & $15.48 b$ & $18.80 \mathrm{~b}$ & $21.10 \mathrm{a}$ & $22.00 \mathrm{a}$ & $25.24 \mathrm{a}$ & $27.00 \mathrm{a}$ & $26.75 a$ \\
\hline & & SS & $15.95 \mathrm{a}$ & $16.62 \mathrm{a}$ & $19.65 \mathrm{a}$ & $21.65 \mathrm{a}$ & $22.50 \mathrm{a}$ & $25.86 \mathrm{a}$ & $26.47 \mathrm{a}$ & $25.75 b$ \\
\hline & \multirow{2}{*}{ Maximum } & NT & $22.52 b$ & $24.71 b$ & $29.15 b$ & $30.95 b$ & $30.95 \mathrm{a}$ & $36.69 \mathrm{a}$ & $35.95 b$ & $33.00 \mathrm{~b}$ \\
\hline & & SS & $23.43 \mathrm{a}$ & $25.62 \mathrm{a}$ & $29.95 \mathrm{a}$ & $32.10 \mathrm{a}$ & $31.70 \mathrm{a}$ & $37.24 \mathrm{a}$ & $37.69 a$ & $34.00 \mathrm{a}$ \\
\hline & \multirow{2}{*}{ Mean } & NT & $18.62 b$ & $20.06 \mathrm{~b}$ & $23.98 b$ & $26.00 \mathrm{~b}$ & $26.45 \mathrm{a}$ & $30.97 \mathrm{a}$ & $31.47 \mathrm{a}$ & $29.88 \mathrm{a}$ \\
\hline & & SS & $19.69 a$ & $21.11 \mathrm{a}$ & $24.80 \mathrm{a}$ & $26.88 \mathrm{a}$ & $27.10 \mathrm{a}$ & $31.55 \mathrm{a}$ & $32.08 \mathrm{a}$ & $29.88 \mathrm{a}$ \\
\hline \multirow{6}{*}{$5-10$} & \multirow{2}{*}{ Minimum } & NT & $11.76 \mathrm{~b}$ & $14.66 \mathrm{~b}$ & $18.65 b$ & $21.50 \mathrm{a}$ & $22.40 \mathrm{a}$ & $25.62 \mathrm{a}$ & $26.84 a$ & $26.65 a$ \\
\hline & & SS & $13.33 \mathrm{a}$ & $15.78 \mathrm{a}$ & $19.35 \mathrm{a}$ & $22.00 \mathrm{a}$ & $22.65 \mathrm{a}$ & $25.76 \mathrm{a}$ & $26.32 \mathrm{a}$ & $25.90 \mathrm{a}$ \\
\hline & \multirow{2}{*}{ Maximum } & NT & $19.19 b$ & $21.09 \mathrm{~b}$ & $26.65 \mathrm{a}$ & $27.60 \mathrm{~b}$ & $29.15 b$ & $34.24 \mathrm{a}$ & $33.95 b$ & $31.75 b$ \\
\hline & & SS & $22.14 \mathrm{a}$ & $21.91 \mathrm{a}$ & $27.05 \mathrm{a}$ & $29.40 \mathrm{a}$ & $30.10 \mathrm{a}$ & $34.71 \mathrm{a}$ & $35.42 \mathrm{a}$ & $33.00 \mathrm{a}$ \\
\hline & \multirow{2}{*}{ Mean } & NT & $15.48 \mathrm{~b}$ & $17.86 \mathrm{~b}$ & $22.33 b$ & $24.55 b$ & $25.78 \mathrm{a}$ & $29.93 a$ & $30.40 \mathrm{a}$ & $29.20 \mathrm{a}$ \\
\hline & & SS & $16.87 \mathrm{a}$ & $18.84 \mathrm{a}$ & $23.20 \mathrm{a}$ & $25.70 \mathrm{a}$ & $26.38 \mathrm{a}$ & $30.24 \mathrm{a}$ & $30.87 \mathrm{a}$ & $29.45 \mathrm{a}$ \\
\hline \multirow{6}{*}{$10-15$} & \multirow{2}{*}{ Minimum } & NT & $15.33 \mathrm{a}$ & $16.26 \mathrm{a}$ & $19.50 \mathrm{a}$ & $22.00 \mathrm{a}$ & $22.80 \mathrm{a}$ & $26.09 a$ & $26.74 a$ & $27.18 \mathrm{a}$ \\
\hline & & SS & $15.95 \mathrm{a}$ & $16.75 \mathrm{a}$ & $20.20 \mathrm{a}$ & $22.35 \mathrm{a}$ & $23.20 \mathrm{a}$ & $25.81 \mathrm{a}$ & $26.05 \mathrm{a}$ & $26.55 \mathrm{a}$ \\
\hline & \multirow{2}{*}{ Maximum } & NT & $21.59 \mathrm{a}$ & $22.10 \mathrm{a}$ & $24.65 \mathrm{a}$ & $26.90 \mathrm{a}$ & $27.95 \mathrm{a}$ & $31.19 \mathrm{a}$ & $32.16 \mathrm{a}$ & $30.90 \mathrm{a}$ \\
\hline & & SS & $20.67 b$ & $21.19 \mathrm{~b}$ & $24.25 \mathrm{a}$ & $26.05 a$ & $28.45 \mathrm{a}$ & $31.14 \mathrm{a}$ & $30.68 b$ & $30.20 \mathrm{a}$ \\
\hline & \multirow{2}{*}{ Mean } & NT & $18.46 \mathrm{a}$ & $19.13 \mathrm{a}$ & $22.43 a$ & $24.45 a$ & $25.38 \mathrm{a}$ & $28.64 a$ & $29.17 \mathrm{a}$ & $29.04 \mathrm{a}$ \\
\hline & & SS & $18.31 \mathrm{a}$ & $18.97 \mathrm{a}$ & $21.90 \mathrm{a}$ & $24.20 \mathrm{a}$ & $25.83 \mathrm{a}$ & $28.48 \mathrm{a}$ & $28.49 \mathrm{a}$ & $28.35 \mathrm{a}$ \\
\hline \multirow{6}{*}{$15-20$} & \multirow{2}{*}{ Minimum } & NT & $15.66 \mathrm{a}$ & $16.29 a$ & $19.26 \mathrm{a}$ & $22.50 \mathrm{a}$ & $23.35 \mathrm{a}$ & $27.14 \mathrm{a}$ & $27.16 \mathrm{a}$ & $27.05 \mathrm{a}$ \\
\hline & & SS & $16.10 \mathrm{a}$ & $16.71 \mathrm{a}$ & $19.65 \mathrm{a}$ & $23.00 \mathrm{a}$ & $23.65 a$ & $27.00 \mathrm{a}$ & $26.79 a$ & $27.55 \mathrm{a}$ \\
\hline & \multirow{2}{*}{ Maximum } & NT & $18.95 \mathrm{a}$ & $19.18 \mathrm{a}$ & $23.25 \mathrm{a}$ & $26.05 \mathrm{a}$ & $26.35 \mathrm{a}$ & $30.05 a$ & $30.11 \mathrm{a}$ & $29.85 \mathrm{a}$ \\
\hline & & SS & $18.00 \mathrm{~b}$ & $18.62 \mathrm{a}$ & $22.75 \mathrm{a}$ & $25.15 \mathrm{~b}$ & $25.95 a$ & $30.00 \mathrm{a}$ & $30.79 a$ & $29.30 \mathrm{a}$ \\
\hline & \multirow{2}{*}{ Mean } & NT & $17.10 \mathrm{a}$ & $17.73 \mathrm{a}$ & $21.26 \mathrm{a}$ & $24.28 \mathrm{a}$ & $24.85 \mathrm{a}$ & $28.60 \mathrm{a}$ & $28.63 \mathrm{a}$ & $28.45 \mathrm{a}$ \\
\hline & & SS & $17.05 \mathrm{a}$ & $17.67 \mathrm{a}$ & $21.20 \mathrm{a}$ & $24.08 \mathrm{a}$ & $24.80 \mathrm{a}$ & $28.52 \mathrm{a}$ & $28.79 \mathrm{a}$ & $28.43 \mathrm{a}$ \\
\hline
\end{tabular}

NT: no tillage, SS: subsoiling tillage; I: March 25, II: April 10, III: April 25, IV: May 10, V: May 25, VI: June 10, VII: July 5, and VIII: July 20. Values between NT and SS for each tillage date followed by different letters are significantly different at $\mathrm{P}<0.05$

\section{Relationship of soil temperature and soil moisture content}

Soil moisture content influences the range of soil temperature under different tillage treatments. For the soil lowest temperature, it showed increasing trend with the soil moisture content increasing at tillage time of I, II, III, IV, and V when the soil temperature was low (Fig. 6). For soil maximum temperature and daily mean temperature, it showed decreased tendency with the soil moisture content increasing at tillage time of IV, V, VI, VII, and VIII. Hence, the soil lowest temperature was easily affected by soil moisture when the soil temperature was very low.

\section{Growth}

Among tillage treatments, SS performed better in improving LAI over the all soil tillage intervals as compared to NT, for both the years (Table 3). During 2012 year, an increasing trend for LAI was recorded for the intervals $\mathrm{I}-\mathrm{V}$, whilst a decrease was 
observed after that for the intervals VI-VIII. This decreasing trend is significantly visible for both the years during VT and R3 growth stages, because of delayed sowing time as compared to initial sowing intervals of $\mathrm{I}-\mathrm{V}$.
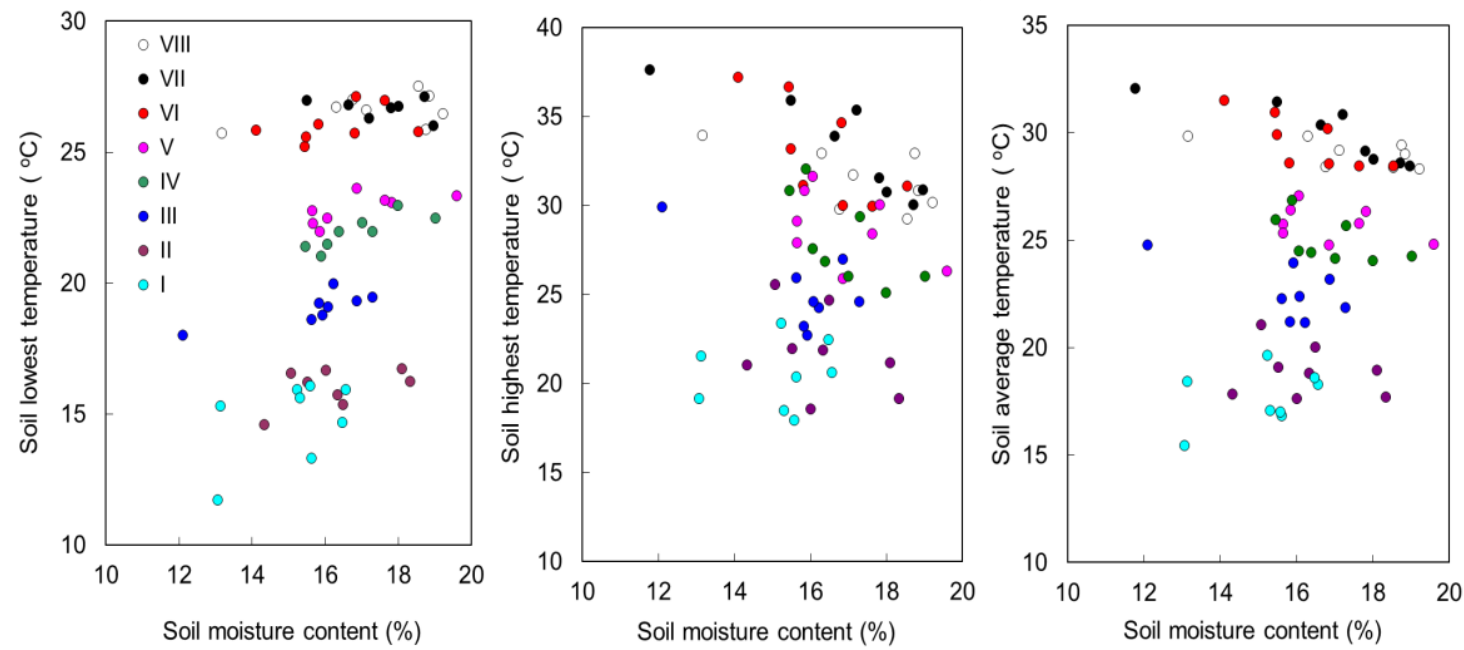

Figure 6. Relationship of soil temperature and soil moisture content at different soil tillage time. I: March 25, II: April 10, III: April 25, IV: May 10, V: May 25, VI: June 10, VII: July 5, and VIII: July 20

Table 3. LAI of maize during crop growth period of maize at eight soil tillage times

\begin{tabular}{|c|c|c|c|c|c|c|c|c|c|c|}
\hline \multirow{2}{*}{ Year } & \multirow{2}{*}{$\begin{array}{c}\text { Growth } \\
\text { stage }\end{array}$} & \multirow{2}{*}{$\begin{array}{l}\text { Soil } \\
\text { tillage }\end{array}$} & \multicolumn{8}{|c|}{ Soil tillage time } \\
\hline & & & $\mathbf{I}$ & II & III & IV & $\mathbf{V}$ & VI & VII & VIII \\
\hline \multirow{8}{*}{2011} & \multirow{2}{*}{ V3 } & NT & $0.05 \mathrm{~b}$ & $0.06 \mathrm{~b}$ & $0.06 \mathrm{~b}$ & $0.07 \mathrm{~b}$ & $0.07 \mathrm{~b}$ & $0.08 \mathrm{~b}$ & $0.10 \mathrm{~b}$ & $0.11 \mathrm{~b}$ \\
\hline & & SS & $0.06 \mathrm{a}$ & $0.07 \mathrm{a}$ & $0.07 \mathrm{a}$ & $0.08 \mathrm{a}$ & $0.09 \mathrm{a}$ & $0.09 \mathrm{a}$ & $0.11 \mathrm{a}$ & $0.12 \mathrm{a}$ \\
\hline & \multirow{2}{*}{ V6 } & NT & $0.43 \mathrm{a}$ & $0.46 \mathrm{~b}$ & $0.49 b$ & $0.58 \mathrm{~b}$ & $0.64 b$ & $0.65 \mathrm{a}$ & $0.65 b$ & $0.75 b$ \\
\hline & & SS & $0.43 \mathrm{a}$ & $0.48 \mathrm{a}$ & $0.51 \mathrm{a}$ & $0.61 \mathrm{a}$ & $0.65 \mathrm{a}$ & $0.65 \mathrm{a}$ & $0.67 \mathrm{a}$ & $0.77 \mathrm{a}$ \\
\hline & \multirow{2}{*}{ VT } & NT & $4.14 \mathrm{~b}$ & $4.33 b$ & $4.50 \mathrm{~b}$ & $4.58 \mathrm{~b}$ & $4.91 \mathrm{~b}$ & $4.77 \mathrm{~b}$ & $3.49 b$ & $3.74 \mathrm{~b}$ \\
\hline & & SS & $4.41 \mathrm{a}$ & $4.69 \mathrm{a}$ & $4.79 \mathrm{a}$ & $4.84 \mathrm{a}$ & $5.16 \mathrm{a}$ & $5.07 \mathrm{a}$ & $3.74 \mathrm{a}$ & $4.03 \mathrm{a}$ \\
\hline & \multirow{2}{*}{ R3 } & NT & $3.14 \mathrm{~b}$ & $3.14 \mathrm{~b}$ & $3.68 \mathrm{~b}$ & $3.88 \mathrm{~b}$ & $4.46 \mathrm{~b}$ & $4.49 b$ & $3.82 b$ & $3.61 \mathrm{~b}$ \\
\hline & & SS & $3.37 \mathrm{a}$ & $3.37 \mathrm{a}$ & $3.82 \mathrm{a}$ & $4.06 \mathrm{a}$ & $4.59 \mathrm{a}$ & $4.57 \mathrm{a}$ & $3.95 \mathrm{a}$ & $3.72 \mathrm{a}$ \\
\hline \multirow{8}{*}{2012} & \multirow{2}{*}{ V3 } & NT & $0.05 \mathrm{~b}$ & $0.06 \mathrm{~b}$ & $0.06 \mathrm{~b}$ & $0.07 \mathrm{~b}$ & $0.07 \mathrm{~b}$ & $0.08 \mathrm{~b}$ & $0.10 \mathrm{~b}$ & $0.11 \mathrm{~b}$ \\
\hline & & SS & $0.06 \mathrm{a}$ & $0.07 \mathrm{a}$ & $0.07 \mathrm{a}$ & $0.08 \mathrm{a}$ & $0.09 \mathrm{a}$ & $0.09 \mathrm{a}$ & $0.11 \mathrm{a}$ & $0.12 \mathrm{a}$ \\
\hline & \multirow{2}{*}{ V6 } & NT & $0.38 b$ & $0.47 \mathrm{~b}$ & $0.58 \mathrm{~b}$ & $0.55 b$ & $0.63 b$ & $0.42 b$ & $0.45 b$ & $0.41 \mathrm{~b}$ \\
\hline & & SS & $0.43 \mathrm{a}$ & $0.52 \mathrm{a}$ & $0.63 \mathrm{a}$ & $0.57 \mathrm{a}$ & $0.67 \mathrm{a}$ & $0.46 \mathrm{a}$ & $0.50 \mathrm{a}$ & $0.44 \mathrm{a}$ \\
\hline & \multirow{2}{*}{ VT } & NT & $4.41 \mathrm{a}$ & $4.92 \mathrm{a}$ & $4.29 b$ & $3.68 \mathrm{~b}$ & $4.56 \mathrm{~b}$ & $4.35 \mathrm{~b}$ & $4.20 \mathrm{a}$ & $3.09 \mathrm{~b}$ \\
\hline & & SS & $4.27 \mathrm{~b}$ & $4.71 \mathrm{~b}$ & $4.36 \mathrm{a}$ & $3.85 \mathrm{a}$ & $4.97 \mathrm{a}$ & $4.69 \mathrm{a}$ & $4.05 \mathrm{~b}$ & $3.33 \mathrm{a}$ \\
\hline & \multirow{2}{*}{ R3 } & NT & $3.91 \mathrm{a}$ & $4.02 \mathrm{~b}$ & $3.89 \mathrm{~b}$ & $3.69 \mathrm{a}$ & $4.15 \mathrm{~b}$ & $4.23 a$ & $3.52 \mathrm{~b}$ & $2.99 \mathrm{a}$ \\
\hline & & SS & $3.90 \mathrm{a}$ & $3.98 \mathrm{a}$ & $3.96 \mathrm{a}$ & $3.42 \mathrm{~b}$ & $4.51 \mathrm{a}$ & $4.13 b$ & $3.92 \mathrm{a}$ & $3.00 \mathrm{a}$ \\
\hline
\end{tabular}

NT: no tillage, SS: subsoiling tillage; I: March 25, II: April 10, III: April 25, IV: May 10, V: May 25, VI: June 10, VII: July 5, and VIII: July 20; Values between NT and SS for each tillage date followed by different letters are significantly different at $\mathrm{P}<0.05$ 
From Figure 7, we could see that SS caused a significant increase in dry weight of shoot and root at 20 days after sowing of maize. For shoot dry weight, the highest value was obtained at soil tillage time of VIII, while the highest increment was obtained at soil tillage time of I under SS (Fig. 7). SS significantly increased shoot dry weight by $13.85 \%(\mathrm{P}<0.01)$ at initial tillage intervals of $\mathrm{I}-\mathrm{IV}$, and by $7.83 \%(\mathrm{P}<0.05)$ at later soil tillage time of V-VIII compared to NT. For root dry weight, the highest value was obtained at soil tillage time of VI, while the highest increment was obtained at soil tillage time of I under SS. Similar to LAI, dry matter accumulation varied across different sowing intervals as well as sampling stages of crop growth period (V3-R3) (Table 4). Maximum value for dry matter was found for SS during sowing interval VI (2011) and V (2012) at R3 sampling stage.

Table 4. Dry matter accumulation of maize during crop growth stages of maize for eight soil tillage times

\begin{tabular}{|c|c|c|c|c|c|c|c|c|c|c|}
\hline \multirow{2}{*}{ Year } & \multirow{2}{*}{$\begin{array}{c}\text { Growth } \\
\text { stage }\end{array}$} & \multirow{2}{*}{$\begin{array}{c}\text { Soil } \\
\text { tillage }\end{array}$} & \multicolumn{8}{|c|}{ Soil tillage time } \\
\hline & & & I & II & III & IV & $\mathbf{V}$ & VI & VII & VIII \\
\hline \multirow{8}{*}{2011} & \multirow[b]{2}{*}{ 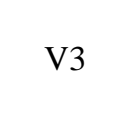 } & NT & $0.34 b$ & $0.47 b$ & $0.61 \mathrm{~b}$ & $0.66 \mathrm{~b}$ & $0.91 \mathrm{~b}$ & $1.16 \mathrm{~b}$ & $1.48 \mathrm{~b}$ & $1.64 \mathrm{~b}$ \\
\hline & & SS & $0.39 \mathrm{a}$ & $0.53 a$ & $0.69 \mathrm{a}$ & $0.75 \mathrm{a}$ & $0.98 \mathrm{a}$ & $1.25 \mathrm{a}$ & $1.60 \mathrm{a}$ & $1.75 \mathrm{a}$ \\
\hline & \multirow{2}{*}{ V6 } & NT & $6.43 b$ & $6.87 \mathrm{~b}$ & $6.40 \mathrm{a}$ & $8.20 \mathrm{a}$ & $8.45 \mathrm{~b}$ & $10.47 b$ & $11.08 \mathrm{~b}$ & $12.90 \mathrm{a}$ \\
\hline & & SS & $8.13 a$ & $9.10 \mathrm{a}$ & $6.97 \mathrm{a}$ & $8.83 a$ & $9.62 \mathrm{a}$ & $11.03 \mathrm{a}$ & $12.49 \mathrm{a}$ & $12.97 \mathrm{a}$ \\
\hline & \multirow{2}{*}{ VT } & NT & $90.03 b$ & $100.80 \mathrm{~b}$ & $116.67 \mathrm{~b}$ & $116.80 \mathrm{~b}$ & $134.53 \mathrm{a}$ & $129.81 \mathrm{~b}$ & $121.87 \mathrm{~b}$ & $116.85 b$ \\
\hline & & SS & $103.20 \mathrm{a}$ & $113.40 \mathrm{a}$ & $133.10 \mathrm{a}$ & 132.99a & $137.67 \mathrm{a}$ & $139.40 \mathrm{a}$ & $137.57 \mathrm{a}$ & $132.99 a$ \\
\hline & & NT & $177.37 \mathrm{~b}$ & $171.60 \mathrm{~b}$ & $186.40 \mathrm{~b}$ & $210.23 b$ & $214.22 \mathrm{~b}$ & $238.47 \mathrm{~b}$ & $186.40 \mathrm{~b}$ & $136.65 \mathrm{~b}$ \\
\hline & & SS & $186.67 \mathrm{a}$ & $186.03 a$ & $198.60 \mathrm{a}$ & $227.07 \mathrm{a}$ & $228.45 \mathrm{a}$ & $252.73 a$ & $198.60 \mathrm{a}$ & $153.70 \mathrm{a}$ \\
\hline \multirow{8}{*}{2012} & \multirow{2}{*}{$\mathrm{V}_{3}$} & NT & $0.34 \mathrm{~b}$ & $0.45 b$ & $0.55 \mathrm{~b}$ & $0.58 \mathrm{~b}$ & $0.98 \mathrm{~b}$ & $1.07 \mathrm{~b}$ & $1.42 \mathrm{~b}$ & $1.75 \mathrm{a}$ \\
\hline & & SS & $0.41 \mathrm{a}$ & $0.51 \mathrm{a}$ & $0.66 \mathrm{a}$ & $0.67 \mathrm{a}$ & $1.07 \mathrm{a}$ & $1.18 \mathrm{a}$ & $1.53 \mathrm{a}$ & $1.79 \mathrm{a}$ \\
\hline & \multirow{2}{*}{ V6 } & NT & $4.10 \mathrm{~b}$ & $4.53 b$ & $5.47 \mathrm{a}$ & $5.23 \mathrm{a}$ & $6.36 \mathrm{~b}$ & $6.60 \mathrm{~b}$ & $8.42 \mathrm{a}$ & $8.75 \mathrm{a}$ \\
\hline & & SS & $5.17 \mathrm{a}$ & $5.25 \mathrm{a}$ & $5.67 \mathrm{a}$ & $5.67 \mathrm{a}$ & $7.35 \mathrm{a}$ & $8.30 \mathrm{a}$ & $8.53 a$ & $9.31 \mathrm{a}$ \\
\hline & \multirow{2}{*}{ VT } & NT & $98.53 b$ & $98.53 b$ & $98.13 b$ & $102.53 b$ & $110.63 b$ & $120.10 \mathrm{a}$ & $94.23 b$ & $86.00 \mathrm{a}$ \\
\hline & & SS & $105.97 \mathrm{a}$ & $102.00 \mathrm{a}$ & $107.97 \mathrm{a}$ & $108.17 \mathrm{a}$ & $115.20 \mathrm{a}$ & $120.83 a$ & $105.30 \mathrm{a}$ & $86.20 \mathrm{a}$ \\
\hline & & NT & $194.53 b$ & $192.53 b$ & $193.97 b$ & $198.10 \mathrm{~b}$ & $214.42 \mathrm{~b}$ & $201.10 \mathrm{~b}$ & $170.20 \mathrm{~b}$ & $145.37 \mathrm{a}$ \\
\hline & & SS & $198.77 \mathrm{a}$ & $194.87 \mathrm{a}$ & $196.80 \mathrm{a}$ & $203.27 \mathrm{a}$ & 219.82a & $204.97 \mathrm{a}$ & $176.63 a$ & $146.13 \mathrm{a}$ \\
\hline
\end{tabular}

NT: no tillage, SS: subsoiling tillage; I: March 25, II: April 10, III: April 25, IV: May 10, V: May 25, VI: June 10, VII: July 5, and VIII: July 20; Values between NT and SS for each tillage date followed by different letters are significantly different at $\mathrm{P}<0.05$

Regardless of soil tillage time, the grain yield of maize was significantly higher under the SS management than no-tillage treatment (Fig. 8). In terms of tillage intervals, the yield of maize was highest in the SS tillage management implemented at VI, but the highest increment of yield was recorded at SS tillage implemented at I. The highest yield potential (YP) of maize was recorded at soil tillage time of V. The potential equivalent was in the range of $71.89-85.10 \%$ at SS, while it was 67.08$81.78 \%$ (range) at NT for tillage times I, II, III, IV, V, VI, and VII, respectively. 


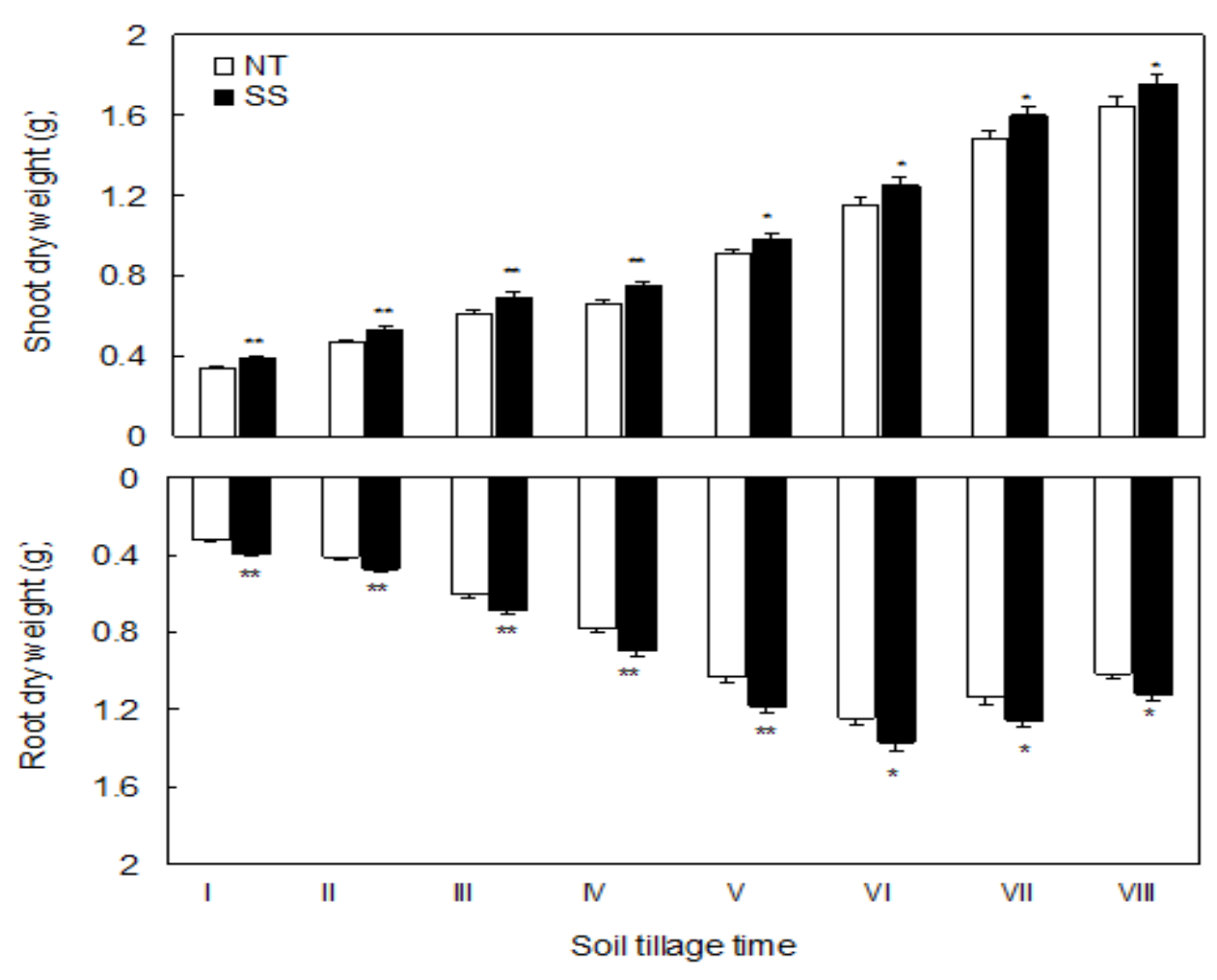

Figure 7. Shoot and root dry matter at 20 days after sowing of maize. NT: no tillage, SS: subsoiling tillage; I: March 25, II: April 10, III: April 25, IV: May 10, V: May 25, VI: June 10,

VII: July 5, and VIII: July 20; ** above the column indicate statistical significance at the $P=0.01$ level within the same soil tillage time; $*$ above the column indicate statistical significance at the $P=0.05$ level within the same soil tillage time

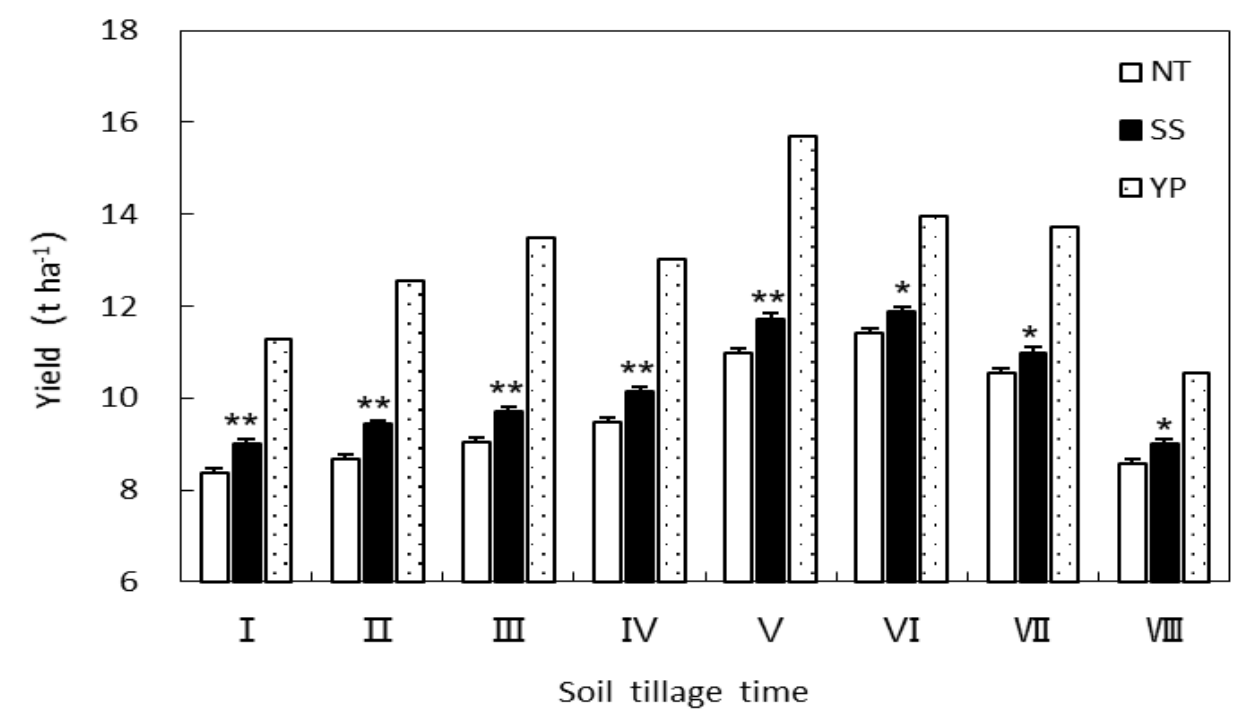

Figure 8. Final grain yield of maize at different soil tillage time. NT: no tillage, SS: subsoiling tillage, YP: yield potential; I: March 25, II: April 10, III: April 25, IV: May 10, V: May 25, VI: June 10, VII: July 5, and VIII: July 20; ** above the column indicate statistical significance at the $P=0.01$ level within the same soil tillage time; * above the column indicate statistical significance at the $P=0.05$ level within the same soil tillage time 


\section{Discussion}

Soil bulk density is an important indicator for soil stiffness (Carter, 1990). Liang et al. (2010) and Liu et al. (2010) found that subsoiling tillage could break the soil hard plough pan and loosen the compacted soil, thereby reducing soil bulk density. Meanwhile because of the dryness and reduced soil viscosity with low soil moisture and temperature, the soil structure could easily be altered (Yang et al., 2013). Our results indicated that SS significantly reduced soil bulk density in 0-30 cm soil layer, especially at the former soil tillage time of $\mathrm{I}-\mathrm{V}$, when compared to no-tillage treatment (Fig. 3). These results are in disagreement with that of Gürsoy et al. (2011). Whereas, Ozpinar and Ozpinar (2015) found a significant decrease in soil bulk density, confirming our results, in top soil due to tillage practices in a five-year experiment at enhancing maize productivity. In 20-30 cm soil layer, because of the destruction of hard plough layer, soil bulk density showed a much significant decrease in present case, promising for sustainable field maize production in China.

Assouline (2006) suggested that, soil can be significantly loosened and the proportion of large pores can be increased significantly under SS tillage. The level of soil porosity is related to intensity of soil tillage (Chen et al., 2009). In present study, the implementation of SS tillage significantly increased soil total porosity in $0-30 \mathrm{~cm}$ soil depth compared with no-tillage treatment (Fig. 4). This improvement can be attributed to the reason that the level and distribution of soil total porosity were significantly affected by the soil bulk density (Raczkowski et al., 2012; Wang et al., 2017).

Soil moisture content is a basic characteristic required for optimum crop growth. Appropriate soil moisture contents could support uptake of crop root and can regulate the dynamics of soil temperature and heat (Karandish and Shahnazari, 2016; Tsironi and Taoukis, 2017). Assouline (2006) showed that SS could improve soil moisture infiltration and storage attributed to the decrease in soil bulk density and increased soil total porosity. Meanwhile the SS tillage could break the continuity of soil pores to prevent the evaporation and reduction of deep soil moisture (Osunbitan, 2005), thereby significantly increasing deep-layer soil moisture contents. However, the SS tillage involves excessive soil ploughing, resulting in relatively higher surface soil moisture evaporation due to more exposure to sunshine (Debaeke and Aboudrare, 2004). And the high soil and air temperature accelerate the surface soil moisture evaporation (Wang et al., 2007). Our study showed the same results that SS significantly increased soil moisture content in 10-30 cm soil depth, while the soil moisture content decreased in 0$10 \mathrm{~cm}$ soil depth compared with no-tillage (Fig. 5). In 10-20 cm soil depth, soil moisture content showed a much significant increase at soil tillage intervals of I, II, III, $\mathrm{V}$, and VI because of the relevant decrease in soil bulk density and increase in soil total porosity due to SS, compared to NT.

In field, seed germination and seedling growth of maize are closely related to soil temperature (Ahmad et al., 2016; Lopushinsky and Max, 1990). Fu et al. (2005) suggested that SS tillage could balance and improve the status of soil temperature through the function of heat preservation on minimum soil temperature conditions and may cause decrease in maximum soil temperature and it could keep the stability of soil temperature, beneficial for the crop growth. In this study, SS tillage increased soil minimum temperature in $0-20 \mathrm{~cm}$ soil depth at soil tillage time of I-VI. For soil maximum temperature and daily mean temperature, the increments were only occurred in $0-10 \mathrm{~cm}$ soil depth at each tillage time (Table 2). Soil temperature jointly determined 
with atmospheric temperature and soil heat conditions are reported to be regulated by soil moisture content and soil porosity (Lakshmi, 2003). Higher soil porosity and moisture content could contribute to heat transfer and storage (Zhang et al., 2009; Wu et al., 2017). For these reasons, the soil minimum temperature showed an increased tendency with increase in soil moisture content, especially at intervals III, IV and V. While the soil maximum and daily mean temperature showed an decreased tendency with increasing soil moisture at later soil tillage time of V, VI, VII and VIII (Fig. 6).

Anand et al. (2012) reported that the decrease in soil penetration resistance, increase in soil moisture and temperature could improve the seedling emergence and growth during early spring under low temperature conditions. Tolon-Becerra et al. (2011) showed that better seedling emergence and growth were achieved under soil with lower compaction, and the relationship between root dry matter and maize yields was strongly positive. In our study, SS significantly increased shoot and root dry matter of maize seedling. Though the highest maize seedling dry matter was obtained at SS tillage time of VI, VII and VIII, which are contrary to the findings of Ge et al. (2013). The reason can be described by effective accumulated temperature, daily mean temperature and accumulated precipitation at respective growth stage of maize (Fig. 2), the maximum increase in maize seedling matter was obtained at tillage intervals of I, II, III, IV and V (Fig. 7). The decrease during former tillage intervals plausibly because of the soil high compaction and low temperature, which may be attributed as main limits for maize seedling growth at March, April, and May (Fig. 1). The improvement in soil compaction and temperature significantly enhanced the maize seedling growth, corroborating the findings of Hoffmann and Kismányoky (2001). The increase in maize yield was significantly improved at SS tillage compared with no-tillage management, which is against the results reported earlier by Mohammadi et al. (2013), and SS also decreased the yield gap between yield and yield potential in our case. Though the highest maize yield was harvested at SS tillage time of V, VI and VII, whilst the highest increment in maize yield was harvested at subsoiling tillage time of I, II, III and IV (Fig. 8), which are contrary to the results of Zhang et al. (2009). Recently, Moinoddini et al. (2017) also found significant positive effects of deep tillage on maize grain yields as compared to no-tillage during the evaluation of different tillage practices and $\mathrm{N}$ application rates under corn-based rotation system. In present case, SS tillage significantly increased soil moisture storage and soil temperature, and improved seedling growth of maize at subsoiling tillage time of I, II, III and IV, as compared no tillage.

Our results indicate that in no-tillage maize growing areas of $\mathrm{HHH}$, soil hydrothermal properties can be improved by implementing the SS in the month of May and June. This improvement reflects in terms of increased soil temperature, moisture content, porosity and the significant decrease in soil bulk density, which in turn is attributed to better seedling growth and early stand establishment with higher shoot/root parameters, and the succeeding plant growth and final dry matter accumulation. The reason can be explained through the maximum accumulated temperature, precipitation (Fig. 2) and the sunshine hours during these months (May, June), which were favorable to improve soil environment and the plant growth under SS tillage implementation, as compared to no-tillage. 


\section{Conclusion}

In Huang-Huai-Hai area of China, SS tillage proved effective in improving soil physical and hydro-thermal properties, and improving maize seedling growth and grain yield, especially when implemented at early time (March, April and May). Compared with no-tillage, subsoiling tillage significantly decreased soil bulk density, and greatly increased soil porosity. SS tillage at early time (especially for May and June) significantly increased the soil moisture storage in 10-30 cm soil depth. Soil minimum temperature $(0-20 \mathrm{~cm})$ and soil maximum temperature $(0-10 \mathrm{~cm})$ significantly increased at SS tillage time of March, April and May. The SS tillage produced maximum dry seedling matter in maize and grain yields compared to no-tillage management, especially when implemented at early time. Because at early sowing of maize, low soil temperature and Spring seasonal drought cause serious hindrance for early stand establishment in $\mathrm{HHH}$. We can conclude that SS tillage could alleviate the soil high compaction and low temperature stress for spring maize, and can alleviate the soil high compaction stress for summer maize. Dry matter accumulation and grain yield was maximum for sowing from May 25 to June 10. It will be of great significance in improving soil environment, seedling growth and grain yield of maize at early sowing with low precipitation and temperature.

Acknowledgements. The research was supported by the National Natural Science Fund 31401952 , National Key R\&D Program of China (2018YFD0200601), Key National Research and Development Programs (2016YFD0300207).

\section{REFERENCES}

[1] Ahmad, W., Noor, M. A., Afzal, I., Bakhtavar, M. A., Nawaz, M. M., Sun, X., Zhou, B., Ma, W., Zhao, M. (2016): Improvement of sorghum crop through exogenous application of natural growth-promoting substances under a changing climate. - Sustainability 8: 1330. http://dx.doi.org/10.3390/su8121330.

[2] Anand, A., Nagarajan, S., Verma, A. P. S., Joshi, D. K., Pathak, P. C., Bhardwaj, J. (2012): Pre-treatment of seeds with static field ameliorates soil water stress in seedlings of maize (Zea mays L.). - Indian J Biochem Biophys. 49: 63-70.

[3] Assouline, S. (2006): Modeling the relationship between soil bulk density and the water retention curve. - Vadose Zone J. 5: 554-563.

[4] Bhaduri, D., Purakayastha, T. J., Chakraborty, D., Chakraborty, K., Singh, M. (2017): Integrated tillage-water-nutrient management effects on selected soil physical properties in a rice-wheat system in the Indian subcontinent. - Arch Agron Soil Sci. DOI: $10.1080 / 03650340.2017 .1332407$.

[5] Certer, M. R. (1990): Relative measures of soil bulk density to characterize compaction in tillage studies on fine sandy loams. - Can J Soil Sci. 70: 425-433.

[6] Chen, J. K., Li, S. J., Zhang, Y., Chen, F., Zhang, H. L. (2009): Characteristics of soil temperature and response to air temperature under different tillage systems - diurnal dynamic of soil temperature and its response to air temperature. - Sci Agric Sinica 42(7): 2592-2600 (in Chinese).

[7] Debaeke, P., Aboudrare, A. (2004): Adaptation of crop management to water-limited environments. - Eur J Agron. 21: 433-446.

[8] Fu, G. Z., Li, C. H., Wang, J. Z., Wang, Z. L., Cao, H. M., Jiao, N. Y., Chen, M. C. (2005): Effects of stubble mulch and tillage managements on soil physical properties and 
water use efficiency of summer maize. - Transactions of the CSAE 21(1): $52-56$ (in Chinese).

[9] Ge, C., Jing, X. Q., Xu, E., Zhao, C. H., Gao, H. M. (2013): Correlation analysis between corn yield and soil physical properties by different subsoiling time and different depth result. - Soil Fert Sci China 3: 15-18 (in Chinese).

[10] Guo, R. P., Lin, Z. H., Mo, X. G., Yang, C. L. (2010): Responses of crop yield and water use efficiency to climate change in the North China Plain. - Agric Water Manag. 97: $1185-1194$.

[11] Gürsoy, S., Sessiz, A., Kiliç, H., Bayram, N. (2011): Tillage system and cotton residue management effects on soil physical and chemical properties of an Anatolian clay loam sown within a wheat-cotton sequence. - Arch Agron Soil Sci. 57(4): 391-400.

[12] Hartmann, P., Zink, A., Fleige, H., Horn, R. (2012): Effect of compaction, tillage and climate change on soil water balance of Arable Luvisols in Northwest Germany. - Soil Tillage Res. 124: 211-218.

[13] Hoffmann, S., Kismányoky, T. (2001): Soil fertility in a long-term fertilizer trial with different tillage systems. - Arch Agron Soil Sci. 46(3-4): 239-250.

[14] Hou, X. Q., Li, R., Jia, Z. K., Han, Q. F., Yang, B. P., Nie, J. F. (2012): Effects of rotational tillage practices on soil structure, organic carbon concentration and crop yields in semi-arid areas of northwest China. - Soil Use Manag. 28: 551-558.

[15] Jabro, J. D., Sainju, U. M., Stevens, W. B., Lenssen, A. W., Evans, R. G. (2009): Longterm tillage influences on soil physical properties under dryland conditions in northeastern Montana. - Arch Agron Soil Sci. 55(6): 633-640.

[16] Ji, B., ZhaoY., Mu, X., Liu, K., Li, C. (2013): Effects of tillage on soil physical properties and root growth of maize in loam and clay in central China. - Plant Soil Environ. 59(7): 295-302.

[17] Karandish, F., Shahnazari, A. (2016): Soil temperature and maize nitrogen uptake improvement under partial root-zone drying irrigation. - Pedosphere 26(6): 872-886.

[18] Lakshmi, V., Jackson, T. J., Zehrfuhs, D. (2003): Soil moisture-temperature relationships: results from two field experiments. - Hydrological Processes 17(15): 30413057.

[19] Liang, J. F., Qi, Q. Z., Jia, X. H., Gong, S. J., Huang, Y. F. (2010): Effects of different tillage managements on soil properties and corn growth. - Ecol Environ Sci. 19(4): 945950 (in Chinese).

[20] Liu, S., He, W. Q., Yan, C. R., Liu, Q. (2010): Effects of different tillage managements on soil physical properties in dryland. - Agri Res Arid Area 28(2): 65-70 (in Chinese).

[21] Liu, X. J., Rong, J. D. (2009): Effects of deep tillage on the properties of soil structure. The Application Technology of Soil and Water Conservation 1: 9-11 (in Chinese).

[22] Lopushinsky, W., Max, T. A. (1990): Effect of soil temperature on root and shoot growth and on budburst timing in conifer seedling transplants. - New For. 4: 107-124.

[23] Lozano-García, B., Parras-Alcántara, L. (2014): Changes in soil properties and soil solution nutrients due to conservation versus conventional tillage in Vertisols. - Arch Agron Soil Sci. 60(10): 1429-1444.

[24] Lu, X., Lu, X., Liao, Y. (2016): Soil $\mathrm{CO}_{2}$ emission and its relationship to soil properties under different tillage systems. - Arch Agron Soil Sci. 62(7): 1021-1032.

[25] Moinoddini, S. S., Koocheki, A., Mahalati, M. N., Borzooei, A. (2017): Tillage and N application effects on crop yield, $\mathrm{N}$ uptake and soil properties in a corn-based rotation. Arch Agron Soil Sci. 63(8): 1150-1162.

[26] Mohammadi, K., Rokhzadi, A., Saberali, S. F., Byzedi, M., Nezhad, M. T. K. (2013): Tillage effects on soil properties and wheat cultivars traits. - Arch Agron Soil Sci. 59(12): 1625-1641.

[27] Nath, A. J., Lal, R. (2017): Effects of tillage practices and land use management on soil aggregates and soil organic carbon in the north Appalachian region, USA. - Pedosphere 27(1): 172-176. 
[28] Noor, M. A. (2017): Nitrogen management and regulation for optimum NUE in maize-A mini review. - Cogent Food \& Agriculture 3(1): 1348214.

[29] Osunbitan, J. A., Oyedele, D. J., Adekalu, K. O. (2005): Tillage effects on bulk density, hydraulic conductivity and strength of a loamy sand soil in southwestern Nigeria. - Soil Tillage Res. 82: 57-64.

[30] Ozpinar, S., Ozpinar, A., (2015): Tillage effects on soil properties and maize productivity in western Turkey. - Arch Agron Soil Sci. 61(7): 1029-1040.

[31] Piao, S. L., Ciais, P., Huang, Y., Shen, Z. H., Peng, S. S., Li, J. S., Zhou, L. P., Liu, H. Y., Ma, Y. C., Ding, Y. H. et al. (2010): The impacts of climate change on water resources and agriculture in China. - Nature 467: 43-51.

[32] Raczkowski, C. W., Mueller, J. P., Busscher, W. J., Bell, M. C., McGraw, M. L. (2012): Soil physical properties of agricultural systems in a large-scale study. - Soil Tillage Re. 119: 50-59.

[33] Tolon-Becerra, A., Tourn, M., Botta, G. F., Lastra-Bravo, X. (2011): Effects of different tillage regimes on soil compaction, maize (Zea mays, L.) seedling emergence and yields in the eastern Argentinean Pampas region. - Soil Tillage Res. 117: 184-190.

[34] Tsironi, T. N., Taoukis, P. S. (2017): Effect of storage temperature and osmotic pretreatment with alternative solutes on the shelf-life of gilthead seabream (Sparus aurata) fillets. - Aquaculture and Fisheries 2: 39-47.

[35] Wang, J., Cai, H. J., Kang, Y. X., Chen, F. (2007): Ratio of soil evaporation to the evapotranspiration for summer maize field. - Transactions of the CSAE 23(4): 17-22 (in Chinese).

[36] Wang, Q. J., Lu, C. Y., Li, H. W., He, J., Khokan, K. S., Rabi, G. R., Liang, Z. H., Qiao, X. D., Li, H., Allen, D. J. M. (2014): The effects of no-tillage with subsoiling on soil properties and maize yield: 12-Year experiment on alkaline soils of Northeast China. Soil Tillage Res. 137: 43-49.

[37] Wang, S., Wang, Z., Gu, J. (2017): Variation patterns of fine root biomass, production and turnover in Chinese forests. - J. Fores. Res. 28: 1185-1194.

[38] Wu, H., Chen, C., Wang, Q., Lin, J., Xue, J. (2017): The biological content of ballast water in China: a review. - Aquaculture and Fisheries 2: 241-246.

[39] Yang, H. S., Dobermann, A., Lindquist, J. L., Walters, D. T., Arkebauer, T. J., Cassman, K. G. (2004): Hybrid-maize: A maize simulation model that combines two crop modeling approaches. - Field Crops Res. 87: 131-154.

[40] Yang, X., Pang, H. C., Li, Y. B., Ren, T. Z., Dong, G. H., Guo, Z. H., Wang, X. J. (2013): Effects of deep rotary subsoiling tillage on the physical properties and crop growth of the Sticky Loamy Soil in north China. - Sci Agric Sinica 46(16): 3401-3412 (in Chinese).

[41] Yin, Y., Gao, J., Liu, Y. (2017): Influence of industrial organizational structure on farming performance of large yellow croaker farmers. - Aquaculture and Fisheries 2: 134-139.

[42] Yu, T. Y., Zhang, X. Y. (2006): Effect of different tillage treatment on black soil water dynamic at tillage layer. - Construction of Modern Agriculture and Farming System 4(1): 511-514 (in Chinese).

[43] Zafar, S. A., Noor, M. A., Waqas, M. A., Wang, X., Shaheen, T., Raza, M., Rahman, M. U. (2018): Temperature Extremes in Cotton Production and Mitigation Strategies. - In: Rahman, M. U., Zafar, Y. (eds.) Past, Present and Future Trends in Cotton Breeding. InTech, Rijeka, Croatia, pp. 65-91 (Chapter 4). dx.doi.org/10.5772/intechopen.74648.

[44] Zhang, S. L., Lars, L., Harald, G., Tong, Y. N., Yang, X. Y., Wang, Q. J. (2009): Effects of mulching and catch cropping on soil temperature, soil moisture and wheat yield on the Loess Plateau of China. - Soil Tillage Res. 102: 78-86.

[45] Zhang, Y. X., Luo, A., Qi, Q. Q., Jiang, Y. M., Cui, H. Q., Cai, Y. (2009): Effect of different cultivation measures on soil temperature in dry areas of west Heilongjiang. Agri Res Arid Areas 27(5): 93-96 (in Chinese). 
[46] Zheng, S. H., Yan, C. R. (2006): The ecophysiological and morphological characteristics of maize in seedling stage under water stress. - Acta Ecol Sinica 26(4): 1138-1143 (in Chinese).

[47] Zhou, L. M., Li, F. M., Jin, S. L., Song, Y. J. (2009): How two ridges and the furrow mulched with plastic film affect soil water, soil temperature and yield of maize on the semiarid Loess Plateau of China. - Field Crops Res. 113: 41-47. 